\section{PCR Primers Containing Stop Codons Reduce the Number of False-Nega- tives during Blue-White Screening}

\section{BioTechniques 34:950-954 (May 2003)}

Many cloning vectors carry the upstream regulatory sequences preceding the genetic information for an N-terminal portion of the $\beta$-galactosidase gene (lacZ), which codes for the so-called $\alpha$ peptide (1). Their multiple cloning sites, which are embedded in the latter, do not disrupt the reading frame but cause an in-frame insertion of a small number of amino acids, which does not interfere with the function of the $\alpha$ - peptide $(2,3)$. Such vectors are transformed in $E$. coli strains with lacZ $\triangle \mathrm{M} 15$ genotype that express the so-called $\alpha$-acceptor or $\omega$-peptide (i.e., the $\mathrm{C}$-terminal portion of the $\beta$-galactosidase). This truncated polypeptide, which typically lacks amino acid residues 11-41 (4), can dimerize but no longer tetramerize. Although neither the $\alpha$-peptide nor the $\omega$-peptide is active by itself, they can associate in order to assemble the active $\beta$-galactosidase holoenzyme. This process has been known as $\alpha$-complementation for more than three decades (5), and its structural basis has been elucidated recently $(6,7)$. Bacterial colonies expressing functional $\beta$-galactosidase are easily recognized on agar plates via the formation of blue colonies in the presence of the chromogenic substrate Xgal (8) or alternative dyes (9). Howev- er, with a certain probability, the insertion of a stretch of foreign DNA into the multiple cloning site leads to the production of a nonfunctional $\alpha$-peptide. As a result, transformed bacterial colonies appear white for two distinct but possibly concomitant reasons. First, inserts disrupt the reading frame because of either frameshift-borne or insert inherent stop signals. Second, especially longer inserts - such as cDNAs, even if cloned in frame and not containing any stops - increase the size of the $\alpha$-peptide in a way that it is rendered incapable of $\alpha$-complementation; this is most likely due to changes in secondary structure.

When SELEX (10-12) (systematic evolution of ligands by exponential enrichment)-derived enriched nucleic acid libraries are cloned before sequencing, it is regularly observed that clearly blue

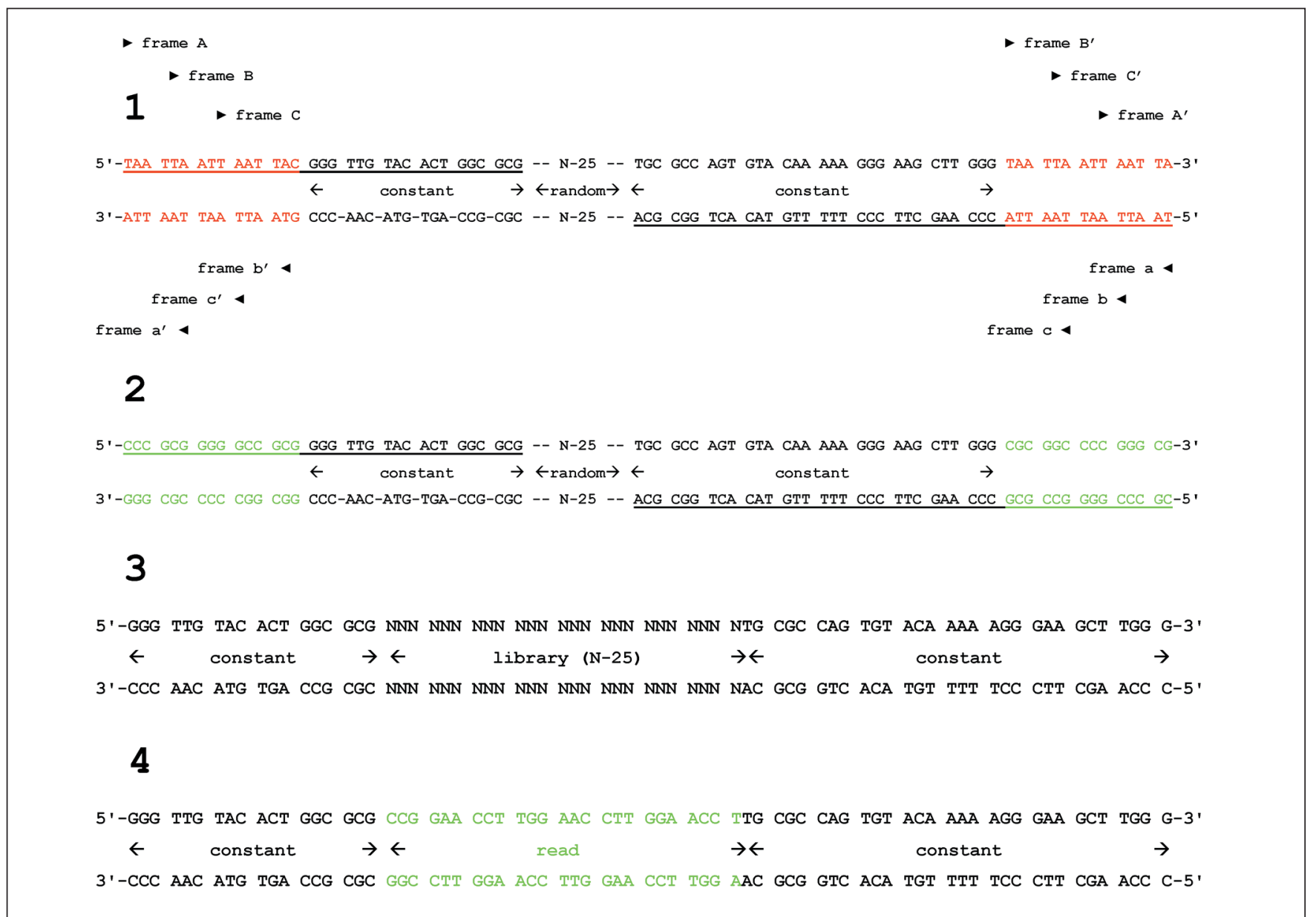

Figure 1. Sequence details of template and primer molecules. 1 and 2 show the template independent sequence details of PCR products amplified with stop and read primers (primers underlined). 3 and 4 show the two template molecules (i.e., the library and the fixed sequence derivative thereof) both as dsDNA. Minus strands only were used as PCR templates. 


\section{Benchmarks}

Table 1. Data of Four Independent Experiments (A-D) Performed with the Different Constructs (I-IV) and Controls (V-VII)

\begin{tabular}{|c|c|c|c|c|c|c|c|c|}
\hline Template & Primers & Exp. & Blue & White & Total & $\%$ White & Construct & No. \\
\hline \multirow{4}{*}{ library } & \multirow[t]{4}{*}{ stop } & A & 24 & 273 & 297 & 92 & \multirow{4}{*}{$=$} & \multirow[t]{4}{*}{ I } \\
\hline & & B & 30 & 219 & 249 & 88 & & \\
\hline & & C & 40 & 247 & 287 & 86 & & \\
\hline & & $D$ & 49 & 514 & 563 & 91 & & \\
\hline \multirow[t]{4}{*}{ library } & \multirow[t]{4}{*}{ read } & $A$ & 158 & 43 & 201 & 21 & \multirow[t]{4}{*}{$=$} & \multirow[t]{4}{*}{ II } \\
\hline & & B & 113 & 24 & 137 & 18 & & \\
\hline & & C & 227 & 52 & 279 & 19 & & \\
\hline & & $D$ & 262 & 61 & 323 & 19 & & \\
\hline fixed & \multirow[t]{2}{*}{ stop } & $A$ & 38 & 547 & 585 & 94 & \multirow{2}{*}{$=$} & \multirow[t]{2}{*}{ III } \\
\hline sequence & & B & 19 & 283 & 302 & 94 & & \\
\hline fixed & \multirow[t]{2}{*}{ read } & A & 368 & 16 & 384 & 4 & \multirow{2}{*}{$=$} & \multirow[t]{2}{*}{ IV } \\
\hline sequence & & B & 145 & 8 & 153 & 5 & & \\
\hline \multirow[t]{2}{*}{ - } & \multirow[t]{2}{*}{ stop } & C & 29 & 3 & 32 & 9 & \multirow[t]{2}{*}{ 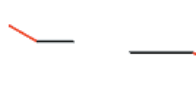 } & \multirow[t]{2}{*}{ V } \\
\hline & & $D$ & 21 & 1 & 22 & 5 & & \\
\hline \multirow[t]{2}{*}{-} & \multirow[t]{2}{*}{ read } & $\mathrm{C}$ & 17 & 23 & 40 & 58 & \multirow[t]{2}{*}{ 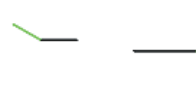 } & \multirow[t]{2}{*}{ VI } \\
\hline & & $D$ & 46 & 36 & 82 & 44 & & \\
\hline \multirow[t]{4}{*}{ - } & \multirow[t]{4}{*}{-} & A & 21 & 2 & 23 & 9 & \multirow[t]{4}{*}{ - } & \multirow[t]{4}{*}{ VII } \\
\hline & & B & 16 & 0 & 16 & 0 & & \\
\hline & & C & 4 & 0 & 4 & 0 & & \\
\hline & & $D$ & 4 & 0 & 4 & 0 & & \\
\hline
\end{tabular}

colonies contain full-length clones as inserts. The explanation for such false negatives lies within the relative shortness of the dsDNA-PCR intermediates of these libraries, which usually range from 50 to 200 nucleotides. If their length is a multiple of three, they are cloned in frame, and happen to contain no stop codons, then a fully functional $\alpha$-peptide is retained.

To circumvent this restriction, we suggest the following simple procedure. After the last round of selection, enriched libraries are amplified with a special set of PCR-primers. Within a stretch of additional bases $5^{\prime}$ adjacent to the fixed regions, these primers contain stop codons, one in each of the three possible reading frames in either direction. The ochre codon UAA was chosen because it was found to be most frequently used in highly expressed genes of $E$. coli (13). It is considered most effective if followed by a uridine base (14). PCR products generated with such a pair of primers will contain one TAAT stop signal in each frame at the $5^{\prime}$ and $3^{\prime}$ end, respectively. No matter in which direction the insert is cloned into the vector, the $\alpha$-peptide will be disrupted and the number of false-negative blue colonies should decrease significantly.

To show the efficiency of this procedure, we designed two sets of primers termed stop and read. The stop primer set contains three TAAT stop signals, one in each frame, within 15 or 14 additional bases, $5^{\prime}$ adjacent to the existing primer binding sites (Figure 1 ). The read primers also contain an additional 15 and 14 bases respectively, but no stop signals. These two sets of primers were used to amplify two different minus-stranded template molecules: a constrained SELEX library, as described by Hamm (15) and a distinct molecule derived thereof. This very library was chosen because it does not contain stop codons in any frame of its constant regions. In the fixed sequence derivative, which was used as a negative control, the N-25 random region was replaced by a defined sequence of equal length that was designed to be free of any stop signals. By combining these materials, a total of four different dsDNA molecules was generated in a 10-cycle two-step PCR between $70^{\circ} \mathrm{C}$ and $94^{\circ} \mathrm{C}$. PCR conditions were $1 \mu \mathrm{M}$ primers, 200 $\mu \mathrm{M}$ dNTPs, 50 fmol ssDNA template (minus strand), $0.5 \mathrm{M}$ betaine, and 2.5 U Taq DNA polymerase in $50 \mu \mathrm{L}$ buffer $(20 \mathrm{mM}$ Tris- $\mathrm{HCl}, \mathrm{pH} 8.4,50$ $\mathrm{mM} \mathrm{KCl}$, and $2.5 \mathrm{mM} \mathrm{MgCl}_{2}$ ). The PCR products were TA-cloned into a pCR $^{\circledR}$ 2.1-TOPO ${ }^{\circledR}$ vector, transformed into One Shot ${ }^{\circledR}$ chemically competent E. coli cells and plated using the TOPO TA cloning kit according to the manufacturer's protocol (Invitrogen). After overnight incubation at $37^{\circ} \mathrm{C}$, blue and white colonies were counted. To estimate the portion of blue colonies that contained a religated vector without insert, vector only was transformed. In analogy, it was attempted to ligate the vector and primers that had been subjected to a no-template control PCR. These experiments (Table 1) led to the following conclusions. The library that had been amplified with read primers, ligated with the vector, and transformed to bacteria (II) yielded approximately $20 \%$ of white colonies, in which the $l a c Z$ reading frame was disrupted because of a statistical distribution of stop codons within the N-25 random region. The same library amplified with stop primers (I) yielded 
approximately $90 \%$ of white colonies. The control template amplified with both primer pairs (III and IV) was used to define the working range of the system. Taking into account that this test system gives rise to varying numbers of false-positive and false-negative colonies of either color that may be transformed with empty vector (VII) or vector with primer artifacts $(\mathrm{V}$ and VI), the number of false-negative blue colonies was reduced by nearly one order of magnitude. Thus, introducing stop codons to small PCR fragments of unknown sequences such as enriched SELEX libraries before cloning can facilitate screening of recombinants. Biased picking of white clones with inserts whose lengths are not multiples of three is efficiently reduced. As an alternative to the procedure presented here, future SELEX libraries can be designed to contain stop codons within their constant regions.

\section{REFERENCES}

1.Messing, J., B. Gronenborn, B. Muller-Hill, and P.H. Hofschneider. 1977. Filamentous coliphage M13 as a cloning vehicle: insertion of a HindII fragment of the lac regulatory region in M13 replicative form in vitro. Proc. Natl. Acad. Sci. USA 74:3642-3646.

2.Brosius, J. 1989. Superpolylinkers in cloning and expression vectors. DNA 8:759-777.

3.Vieira, J. and J. Messing. 1991. New pUCderived cloning vectors with different selectable markers and DNA replication origins. Gene 100:189-194.

4.Langley, K.E., M.R. Villarejo, A.V. Fowler, P.J. Zamenhof, and I. Zabin. 1975. Molecular basis of $\beta$-galactosidase $\alpha$-complementation. Proc. Natl. Acad. Sci. USA 72:12541257

5.Ullmann, A., F. Jacob, and J. Monod. 1967. Characterization by in vitro complementation of a peptide corresponding to an operatorproximal segment of the $\beta$-galactosidase structural gene of Escherichia coli. J. Mol. Biol. 24:339-343.

6.Jacobson, R.H., X.J. Zhang, R.F. DuBose, and B.W. Matthews. 1994. Three-dimensional structure of $\beta$-galactosidase from $E$. coli. Nature 369:761-766.

7.Juers, D.H., R.H. Jacobson, D. Wigley, X.J. Zhang, R.E. Huber, D.E. Tronrud, and B.W. Matthews. 2000. High resolution refinement of $\beta$-galactosidase in a new crystal form reveals multiple metal-binding sites and provides a structural basis for $\alpha$-complementation. Protein Sci. 9:1685-1699.

8.Horwitz, J.P., J. Chua, R.J. Curby, A.J. Tomson, M.A. DaRooge, B.E. Fisher, J. Mauricio, and I. Klundt. 1964. Substrates for cytochemical demonstration of enzyme activity. I. Some substituted 3 -indolyl- $\beta$-Dgalactopyranosides. J. Med. Chem. 7:574.

9.Heuermann, K. and J. Cosgrove. 2001 SGal: an autoclavable dye for color selection of cloned DNA inserts. BioTechniques 30:11421147.

10.Robertson, D.L. and G.F. Joyce. 1990. Selection in vitro of an RNA enzyme that specifically cleaves single-stranded DNA. Nature 344:467-468

11.Ellington, A.D. and J.W. Szostak. 1990. In vitro selection of RNA molecules that bind specific ligands. Nature 346:818-822.

12. Tuerk, C. and L. Gold. 1990. Systematic evolution of ligands by exponential enrichment: RNA ligands to bacteriophage T4 DNA polymerase. Science 249:505-510.

13.Nakamura, Y., T. Gojobori, and T. Ikemura. 2000. Codon usage tabulated from international DNA sequence databases: status for the year 2000. Nucleic Acids Res. 28:292.

14.Tate, W.P. and S.A. Mannering. 1996. Three, four or more: the translational stop signal at length. Mol. Microbiol. 21:213-219.

15.Hamm, J. 1996. Characterisation of antibody-binding RNAs selected from structurally constrained libraries. Nucleic Acids Res. 24:2220-2227.

We are grateful to Christian Lange, Sandra Stark, Dirk Eulberg, and Axel Vater for helpful discussions and critical reading of the manuscript. Address correspondence to Dr. Sven Klussmann, NOXXON Pharma AG, Max-Dohrn-Strasse 8-10, D-10589 Berlin, Germany. e-mail: sklussmann@ noxxon.net

Received 10 January 2003; accepted 25 February 2003.

\section{Josmar Langner and Sven Klussmann NOXXON Pharma AG Berlin, Germany}

\section{Improved Method for the Construction of Full- Length Enriched cDNA Libraries}

BioTechniques 34:954-957 (May 2003)

Reverse transcription of cDNA from mRNA is one of the most widely used applications in molecular biology. The study of transcribed genes has greatly depended on the reverse transcriptase's synthesis of cDNA molecules for library construction, RT-PCR, and for use as DNA probes. Unfortunately, the inherent lack of processivity of reverse transcriptase and the presence of mRNA secondary structure reduce the enzyme's efficiency at synthesizing full-length cDNAs. Various approaches have been undertaken to improve the synthesis of full-length cDNAs, which in this report are defined as DNA molecules covering the transcription initiation to the polyadenylation sites of template mRNAs. Most notably, a deletion derivative of the cloned Moloney murine leukemia virus (MMLV) reverse transcriptase gene that eliminates the RNase $\mathrm{H}$ component of the reverse transcriptase is now commercially available (1). When used in conjunction with high-quality, size-fractionated RNAs, the modified reverse transcriptase has been reported to be highly efficient at creating full-length cDNAs (2). Other approaches have been aimed at heat denaturation of mRNA secondary structure during reverse transcription. Reactions performed at elevated temperatures have been accomplished with the use of thermophilic enzymes (3) or with chemical additives to thermostabilize MMLV (4). Yet, despite these improvements, many cDNA molecules are still not full length. This is possibly because reverse transcriptases have high error rates and are likely to incorporate wrong nucleotides during reverse transcription. After a mis-insertion, MMLV, for example, will only resume faithful reverse transcription $50 \%$ of the time (5). Therefore, additional steps are needed to select specifically for full-length cDNA molecules. Examples include the use of an immobilized mRNA cap-binding protein (6), 